\title{
Retrospective Analysis of Clinicopathological
}

\section{Features and Prognosis of Gynecological Small-Cell Carcinoma}

\author{
Li Pang $\mathbb{D}^{\prime}$ \\ Hui Yang \\ Yuer Ning' \\ Chunyu Zheng ${ }^{2}$ \\ 'Department of Obstetrics and \\ Gynecology, Shengjing Hospital of China \\ Medical University, Shenyang, I 10004 \\ Liaoning, People's Republic of China; \\ ${ }^{2}$ Department of Chinese Journals of \\ Practical Medicine, China Medical \\ University, Shenyang, I I000I, Liaoning, \\ People's Republic of China
}

Correspondence: Chunyu Zheng No.77 Puhe Road, Shenyang North NewArea, Shenyang City, I I000I, Liaoning Province, People's Republic of China

Tel +86 24-|89009|| 992

$\mathrm{Fax}+86$ 24-23866478

Email147509892@I63.com
Purpose: Although rare, small-cell neuroendocrine carcinoma of the gynecologic tract (SCNCGT) is associated with poor prognosis. We analyzed the clinical characteristics, pathological features, treatment strategies, and prognosis in patients with SCNCGT.

Patients and Methods: We performed a retrospective data analysis of 34 patients with SCNCGT diagnosed and treated at our hospital between 2006 and 2018. Medical records were reviewed for pathological features, treatment methods, and outcomes of this disease.

Results: We included 34 patients who had small-cell neuroendocrine carcinoma of the endometrium (SCNCE; 7), ovary (SCNCO; 7), and cervix (SCNCC; 20). All patients with SCNCE underwent comprehensive surgery and six received postoperative chemotherapy. All patients with SCNCO received chemotherapy after surgery; six underwent comprehensive surgery and one underwent treatment only in the pelvic cavity. Sixteen patients with SCNCC underwent radical surgery and received chemotherapy, two of whom received simultaneous radiotherapy. The remaining four patients with SCNCC underwent comprehensive chemotherapy and radiation therapy. Among the 34 patients, 11 had vascular metastases, 15 had lymph node metastases, and seven exhibited positive margins. The median overall survival time among all patients was 23.18 months (range: 3-66 months). Death occurred in 18 cases $(52.94 \%)$. Recurrence was observed in 13 cases $(38.24 \%)$. The average time to recurrence was 15.78 months following treatment (range: 2-30 months). All 34 patients were evaluated for neuroendocrine markers. The immunohistochemical positive rates of synaptophysin, CD56, and chromogranin A were $73.5 \%, 64.7 \%$, and $55.9 \%$, respectively.

Conclusion: The rates of metastasis and recurrence are high, and prognosis remains poor, even among patients with early-stage SCNCGT. Our data may aid in the development of reference standards for diagnosis and treatment.

Keywords: comprehensive treatment, gynecologic tract, malignant tumor, metastasis, smallcell carcinoma

\section{Introduction}

Small-cell carcinoma is an argyrophilic form of neuroendocrine carcinoma that can synthesize and secrete amine and polypeptide hormones, thereby exerting the effects of neurotransmitters, hormones, and paracrine factors. Cancer cells originate from the neuroendocrine cells and are widely distributed throughout the human body. Therefore, in addition to traditional endocrine organs, small-cell carcinomas can occur in the lungs, gastrointestinal tract, liver, prostate, bladder, cervix, and ovaries. ${ }^{1}$ Given its rarity, primary small-cell neuroendocrine carcinoma of the 
gynecologic tract (SCNCGT) poses a significant clinical challenge. Although the age at the onset of SCNCGT varies widely, previous studies have reported that the age at the onset of small-cell neuroendocrine carcinoma of the endometrium (SCNCE) is approximately 10 years greater than that at the onset of normal endometrial cancer. It was also reported that most women with small-cell carcinoma of the uterus had already given birth. The average age at onset after giving birth two or more times is approximately 60 years, which is significantly higher than the average age at the onset of adenocarcinoma. ${ }^{2}$ The clinical manifestations of SCNCGT are nonspecific, mainly including abnormal vaginal bleeding, contact vaginal bleeding, and abdominal pain. Some patients may also experience endocrine symptoms, such as Cushing's syndrome, hypercalcemia, and abnormal secretion of antidiuretic hormone. ${ }^{3-5}$ These manifestations occur because of neuroendocrine disorders caused by increases in the levels of hormones or serum antibodies produced by tumor cells.

The incidence of SCNCGT is low, accounting for approximately $2 \%$ of all gynecological malignancies. SCNCGT most commonly occurs in the cervix, followed by the ovaries and endometrium. A few reports in the literature state that SCNCGT is highly malignant, exhibits aggressive biological behaviors, has a poor prognosis, and has few long-term survival cases; ${ }^{6}$ most of the studies are case reports and only a few cases have been analyzed. Primary SCNCGT exhibits histopathological and biological characteristics similar to those of lung small-cell carcinoma, manifesting as a single small round cell of the same size and shape with less cytoplasm and a hyperchromatic nucleus. Primary SCNCGT is also associated with a more frequent mitotic morphology and early vascular metastasis. ${ }^{7-9}$

Currently, immunohistochemical techniques are required for an auxiliary diagnosis of SCNCGT, and definitive diagnosis remains challenging. Although the need for surgical intervention is based on the International Federation of Gynecology and Obstetrics (FIGO) criteria for gynecological cancer, there are no standardized treatment methods. To improve the understanding of this uncommon malignancy and provide diagnostic and therapeutic guidance for clinicians, we retrospectively analyzed the clinical/pathological features of SCNCGT, treatment methods, and prognosis among 34 patients treated at a single center. The results are expected to provide references for clinicians to diagnose and treat SCNCGT.

\section{Patients and Methods}

This study included 34 patients who received an initial pathological diagnosis of SCNCGT and underwent treatment at the ShengJing Hospital of China Medical University between December 31, 2006, and December 31, 2018. Although we originally selected 39 patients with confirmed SCNCGT during this 12-year period, five patients were lost to follow-up. Among the remaining 34 patients with complete follow-up data, SCNCE, small-cell neuroendocrine carcinomas of the ovary (SCNCO), and cervix (SCNCC) were noted in seven, seven, and 20 patients, respectively. The median follow-up duration was 36 months (overall range: 3-66 months).

We retrospectively analyzed data including initial patient age, clinical symptoms, imaging findings (ultrasound, computed tomography [CT], magnetic resonance imaging [MRI]), examination findings (tumor markers), diagnoses (clinical/pathological), treatments, recurrence rate, time to recurrence, and location. We also reviewed histopathological and immunohistochemical characteristics. All histopathological examinations were performed by two pathologists. For patients with ovarian cancer and endometrial cancer, the clinical stage was determined in accordance with 2009 FIGO standards. ${ }^{10,11}$ For patients with cervical cancer, clinical staging was performed in accordance with the 2018 FIGO standards. ${ }^{12,13}$ Diagnoses of SCNCGT were made based on the World Health Organization criteria for neuroendocrine tumors. ${ }^{14}$ Disease-free survival (DFS) was calculated based on the time from radical hysterectomy $(\mathrm{RH})$ to cancer recurrence or to the final follow-up. Overall survival (OS) was calculated based on the time from RH to death or to the final follow-up.

This retrospective study was approved by the Ethics Committee of ShengJing Hospital of China Medical University. All patients provided informed written consent. This study was conducted in accordance with the Declaration of Helsinki.

Cumulative DFS and OS rates were calculated using the Kaplan-Meier method, and the survival curves were compared using Log rank tests. All data were analyzed using SPSS 23.0 software (IBM Corp., Armonk, NY USA). Kaplan-Meier survival curves were drawn using "GraphPad Prism (8.3.0 GraphPad Software, San Diego, CA, USA). $P$ values $<0.05$ were considered statistically significant. 


\section{Results}

\section{Clinical Manifestations}

The average age of the 34 included patients with SCNCGT was 43 years (range: 36-82 years). Of these patients, seven (20.6\%) had SCNCE, seven had SCNCO (20.6\%), and $20(58.8 \%)$ had SCNCC. FIGO stages were IA $(n=2)$, IB $(n=1)$, IIA $(n=1)$, IIIC $(n=2)$, and IVB $(n=1)$ in the seven patients with SCNCE (inguinal lymph nodes positive). In the seven patients with SCNCO, FIGO stages were IA ( $\mathrm{n}=2)$, IIA ( $\mathrm{n}=1)$, IIIC $(\mathrm{n}=3)$, and IV $(\mathrm{n}=1$, metastasis to the liver parenchyma). In the 20 patients with SCNCC, FIGO stages were IB1 $(n=3)$, IB2 $(n=2)$, IB3 $(\mathrm{n}=4)$, IIA $(\mathrm{n}=7), \operatorname{IIB}(\mathrm{n}=1), \operatorname{IIIB}(\mathrm{n}=1)$, and IVB $(\mathrm{n}=2$, bladder metastasis). Overall, 24 patients exhibited FIGO stages I-II (70.6\%), while 10 exhibited FIGO stages III-IV.

Gross tumor measurements were available in all 34 cases, with tumor dimensions ranging from $2 \mathrm{~cm}$ to $17 \mathrm{~cm}$. In patients with SCNCE and SCNCO, CA125 levels ranged from 13.5 to $184.2 \mathrm{U} / \mathrm{mL}$ (median: $56.2 \mathrm{U} /$ $\mathrm{mL}$ ) and from 22.56 to $970 \mathrm{U} / \mathrm{mL}$ (median: $205.75 \mathrm{U} / \mathrm{mL}$ ), respectively. Squamous cell carcinoma (SCC) levels ranged from 0.3 to $1.4 \mathrm{ng} / \mathrm{mL}$ (median: $0.8 \mathrm{ng} / \mathrm{mL}$ ). Vascular and lymph node metastases were noted in 11 and 15 patients, respectively. Positive margins were identified in seven patients.

Clinical symptoms were nonspecific in most patients. Varying degrees of vaginal bleeding were observed among the seven patients with SCNCE, although only one of them experienced bleeding with abdominal distension. Two of the seven patients with SCNCO had no symptoms and were diagnosed based on the results of routine physical examinations. Four other patients with SCNCO experienced varying degrees of abdominal distension and abdominal pain, while one patient visited the clinic due to abdominal distension and difficulty with bowel movements. Among the 20 patients with SCNCC, four experienced vaginal bleeding, 13 experienced bleeding during intercourse, and three were asymptomatic. No significant increases in SCC were observed in patients with SCNCC, and CA125 levels were elevated in only one patient with SCNCO. The initial clinical characteristics of the 34 included patients with SCNCGT are presented in Table 1.

\section{Immunohistochemical Characteristics}

Upon initial diagnosis, the assessments of neuroendocrine markers were performed in all 34 patients. Rates of immunohistochemical positivity for synaptophysin, CD56, chromogranin A, KI67, neuron-specific enolase (NSE), and creatine kinase (CK) were $73.5 \%, 64.7 \%$, $55.9 \%, 52.9 \%, 50 \%$, and $44.1 \%$, respectively. The rates of positivity were lower for NSE and synaptophysin than those for CD56, although the positive rate for synaptophysin remained higher than that for CD56. All tumors were positive for at least one neuroendocrine marker. For the patients, two were positive for only one neuroendocrine marker, five were positive for two neuroendocrine markers, and 27 were positive for all three markers of neuroendocrine differentiation. The results of immunohistochemical staining are summarized in Table 2, while the pathological images are presented in Figure 1.

\section{Treatments}

Of the 34 patients included in this study, 29 underwent surgery. Among the five patients who did not undergo surgery, an 84-year-old patient was pathologically diagnosed with SCNCO after abdominal puncture. The enhanced CT scan showed metastasis in the liver parenchyma (stage IV), and first-line adjuvant chemotherapy was administered. Four patients with SCNCC (IIB-IVB) who did not undergo surgery received concurrent radiotherapy and chemotherapy (RTC).

Of the seven patients with SCNCE, two underwent laparotomic extra-fascial hysterectomy $(\mathrm{EFH})$, two underwent laparotomic EFH + adnexectomy + pelvic lymph node dissection + para-aortic lymph node resection $(\mathrm{EFH}$ $+\mathrm{AD}+\mathrm{LN}+\mathrm{Pa}-\mathrm{LN})$, and one received supplemental chemotherapy with three cycles of etoposide + cisplatin (EP) post-surgery. Chemotherapy was discontinued in this patient due to platelet inhibition. Three patients received four cycles of EP, and three underwent laparotomic cytoreductive surgery (CS). Two patients undergoing CS received six cycles of EP and one patient undergoing CS received one cycle of EP.

Of the seven patients with SCNCO, one underwent unilateral $\mathrm{AD}$, and the postoperative pathological examination confirmed the diagnosis of SCNCO. An 85-yearold patient declined supplementary surgery due to age and received chemotherapy. Two patients underwent staged surgery for ovarian cancer (hysterectomy + double $\mathrm{AD}+$ omentum resection + appendectomy $+\mathrm{LN}$ $+\mathrm{Pa}-\mathrm{LN})$ and six cycles of postoperative EP. In one patient, an adnexal mass was found at 17 weeks of gestation, following which labor was induced. Four 
Table I Initial Clinical Characteristics of Small-Cell Neuroendocrine Carcinoma of Gynecologic Tract for 34 Patients

\begin{tabular}{|c|c|c|c|}
\hline Characteristics & SCNCE & SCNCO & SCNCC \\
\hline & $(n=7), n(\%)$ & $(n=7), n(\%)$ & $(n=20), n(\%)$ \\
\hline \multicolumn{4}{|l|}{ Age } \\
\hline$<55$ years & $6(85.7)$ & $4(57.2)$ & $2(10.0)$ \\
\hline$\geq 55$ years & $\mathrm{I}(14.3)$ & $3(42.8)$ & $18(90.0)$ \\
\hline \multicolumn{4}{|l|}{ FIGO stage } \\
\hline 1 & $3(42.8)$ & $2(28.6)$ & $9(45.0)$ \\
\hline$\|$ & $\mathrm{I}(14.3)$ & $\mathrm{I}(14.3)$ & $8(40.0)$ \\
\hline III & $2(28.6)$ & $3(42.8)$ & $I(5.0)$ \\
\hline IV & $\mathrm{I}(14.3)$ & $\mathrm{I}(14.3)$ & $2(10.0)$ \\
\hline \multicolumn{4}{|l|}{ Symptoms } \\
\hline Vaginal bleeding & $6(85.7)$ & $0(0.0)$ & $4(20.0)$ \\
\hline $\begin{array}{l}\text { Abdominal distension and } \\
\text { abdominal pain }\end{array}$ & $\mathrm{I}(14.3)$ & $4(57.2)$ & $0(0.0)$ \\
\hline $\begin{array}{l}\text { Abnormal bowel } \\
\text { movements }\end{array}$ & $0(0.0)$ & $\mathrm{I}(14.2)$ & $0(0.0)$ \\
\hline Asymptomatic & $0(0.0)$ & $2(28.6)$ & $3(15.0)$ \\
\hline Bleeding during intercourse & $0(0.0)$ & $0(0.0)$ & $13(65.0)$ \\
\hline \multicolumn{4}{|l|}{ Tumor diameter } \\
\hline$\leq 4 \mathrm{~cm}$ & $5(71.4)$ & $0(0.0)$ & $7(35.0)$ \\
\hline $5-9 \mathrm{~cm}$ & $2(28.6)$ & $2(28.6)$ & $13(65.0)$ \\
\hline$\geq 10 \mathrm{~cm}$ & $0(0.0)$ & $5(7 \mid .4)$ & $0(0.0)$ \\
\hline \multicolumn{4}{|l|}{ Lymphovascular infiltration } \\
\hline Y & $3(42.8)$ & $2(28.6)$ & $6(30.0)$ \\
\hline$N$ & $4(57.2)$ & $5(71.4)$ & 14(70.0) \\
\hline \multicolumn{4}{|l|}{ Lymph node metastasis } \\
\hline $\mathrm{Y}$ & $3(42.8)$ & $4(57.2)$ & $8(40.0)$ \\
\hline N & $4(57.2)$ & $3(42.8)$ & $12(60.0)$ \\
\hline \multicolumn{4}{|l|}{ CAI $25(\mathrm{U} / \mathrm{mL})$} \\
\hline$\geq 1000$ & $0(0.0)$ & $0(0.0)$ & - \\
\hline$<1000$ & $7(100.0)$ & $7(100.0)$ & - \\
\hline \multicolumn{4}{|l|}{$\mathrm{SCC}(\mathrm{ng} / \mathrm{mL})$} \\
\hline$\geq 1.5$ & - & - & $0(0.0)$ \\
\hline$<1.5$ & - & - & $20(100.0)$ \\
\hline \multicolumn{4}{|l|}{ Incisal margin } \\
\hline$P$ & $2(28.6)$ & $\mathrm{I}(14.3)$ & $4(20.0)$ \\
\hline $\mathrm{N}$ & $5(71.4)$ & $6(85.7)$ & $16(80.0)$ \\
\hline
\end{tabular}

Abbreviations: SCNCE, small-cell neuroendocrine carcinomas of the endometrium; SCNCO, small-cell neuroendocrine carcinomas of the ovary; SCNCC, smallcell neuroendocrine carcinomas of the cervix; $Y$, yes; N, no; P, positive; $N$, negative.

days after the labor, the patient underwent laparotomic $\mathrm{CS}$ for ovarian cancer and four postoperative cycles of EP; due to the degree of myelosuppression (IV), the patient refused further chemotherapy. Two patients underwent CS and six cycles of EP. Two patients underwent six cycles of bleomycin + etoposide + cisplatin (BEP), one of whom was diagnosed with FIGO stage
IVB gynecological cancer with metastasis to the liver parenchyma. Due to advanced age, the patient underwent a peritoneal biopsy, one cycle of BEP, and one cycle of EP.

Of the 20 patients with SCNCC, 16 underwent RH $+\mathrm{LN}$ (five underwent laparoscopic surgery, 11 underwent laparotomic surgery). Of these 16 patients, 10 simultaneously underwent $\mathrm{Pa}-\mathrm{LN}$, nine menopausal patients simultaneously underwent double $\mathrm{AD}$, and seven nonmenopausal patients underwent double ovarian suspension. One patient with stage IIA gynecological cancer received three cycles of docetaxel + cisplatin (TP) neoadjuvant chemotherapy preoperative, followed by RH + LN and two cycles of TP postoperatively. Paclitaxel/carboplatin (TC) chemotherapy, TC + RT, TC + biological treatment, TC + EP, TP + RT, and single EP chemotherapy were utilized in four, two, one, one, one, and six patients, respectively. RTC were performed in four patients with FIGO staging greater than IIB. Further details are included in Table 3.

\section{Follow-Up and Prognosis}

Follow-up was performed until December 31, 2018, by interviewing the 34 patients included in this study via telephone. Among the 34 patients with SCNCGT, the median OS was 23.18 months (range: 3-66 months). One patient with stage IV SCNCE had an OS of three months. Death occurred in 18 cases (52.94\%), and recurrence was noted in 13 (38.24\%). On average, recurrence was observed 15.78 months following treatment (range: 2-30 months). Among the patients with SCNCE, the median OS was 19 months (range: 2-34 months), and 19.28 months (range: 4-36 months) for the patients with SCNCO. Among the patients with SCNCC, the median OS was 26 months (range: 5-66 months). The median DFS among all 34 patients was 20 months (range: 1-66 months). For the patients with SCNCE, the median DFS was 15.81 months (range: 1-26 months), and 14.71 months (range: 3-30 months) for patients with SCNCO. Among the patients with SCNCC, the median DFS was 24 months (range: 566 months). The two-year DFS and OS rates of all 34 patients were $48.8 \%$ and $58.2 \%$, respectively (Figure $2 \mathrm{~A}$ and B). The two-year DFS rates were $42.9 \%, 47.6 \%$, and $63.1 \%$ in the SCNCE, SCNCO, and SCNCC groups, respectively ( $P=0.131$; Figure 2C). The two-year OS rates in these groups were $34 \%, 27 \%$, and $55.7 \%$, respectively $(P=0.132$; Figure 2D). Although the difference was 
Table 2 Immunohistochemical Staining Results in Small-Cell Neuroendocrine Carcinoma of Gynecologic Tract for 34 Patients

\begin{tabular}{|c|c|c|c|c|c|c|c|}
\hline $\begin{array}{l}\text { NE } \\
\text { Markers }\end{array}$ & $\begin{array}{c}\text { SCNCE } \\
(n=7)\end{array}$ & $\begin{array}{c}\text { Positive Rate } \\
\%\end{array}$ & $\begin{array}{c}\text { SCNCO } \\
(n=7)\end{array}$ & $\begin{array}{c}\text { Positive Rate } \\
\%\end{array}$ & $\begin{array}{c}\text { SCNCC } \\
(n=20)\end{array}$ & $\begin{array}{c}\text { Positive Rate } \\
\%\end{array}$ & $\begin{array}{c}\text { Total positive } \\
\text { Rate } \%\end{array}$ \\
\hline CD 56 & 3 & 42.9 & 6 & 85.7 & 13 & 65 & 64.7 \\
\hline P63 & I & $\mathrm{N}$ & I & $\mathrm{N}$ & 14 & $\mathrm{~N}$ & 5.8 \\
\hline KI67 & 2 & 28.6 & 4 & 57.1 & 12 & 60 & 52.9 \\
\hline SYN & 4 & 57.1 & 5 & 71.4 & 17 & 85 & 73.5 \\
\hline NSE & 3 & 42.9 & 3 & 42.9 & 11 & 55 & 50 \\
\hline CK & 2 & 28.6 & 3 & 42.9 & 10 & 50 & 44.1 \\
\hline $\mathrm{CgA}$ & 3 & 42.9 & 3 & 42.9 & 13 & 65 & 55.9 \\
\hline TTF-I & 3 & 42.9 & 4 & 57.1 & I & 5 & 23.5 \\
\hline P40 & I & $\mathrm{N}$ & 4 & $\mathrm{~N}$ & 13 & $\mathrm{~N}$ & 14.7 \\
\hline
\end{tabular}

Abbreviations: NE, neuroendocrine; SCNCE, small-cell neuroendocrine carcinomas of the endometrium; SCNCO, small-cell neuroendocrine carcinomas of the ovary; SCNCC, small-cell neuroendocrine carcinomas of the cervix; N, negative.

not statistically significant, the two-year DFS and OS rates were higher in the SCNCC group than in the SCNCE and SCNCO groups $(P=0.131, P=0.132)$.

The two-year DFS rates were $50.9 \%$ and $23.0 \%$ in the early-stage and advanced-stage group, respectively $(P=0.265$; Figure 2E). The two-year OS rates were $60.0 \%$ and $39.8 \%$ in the early-stage and advanced-stage groups, respectively ( $P=0.159$; Figure $2 \mathrm{~F})$. Although the difference was not statistically significant, the two-year DFS and OS rates were higher in the early-stage group than in the advanced-stage group ( $P=0.265, P=0.159)$.

\section{Discussion}

We included 34 patients who had SCNCGT. All the patients with SCNCE underwent comprehensive surgery. All the patients with SCNCO received chemotherapy after surgery. Sixteen patients with SCNCC underwent radical surgery and received chemotherapy, two of whom received simultaneous radiotherapy. The remaining four patients with SCNCC underwent comprehensive chemotherapy and radiation therapy. Our research shows that women with SCNCGT had a poor overall survival rate. The median overall survival time among all patients was 23.18 months. Death was observed in 18 cases. Recurrence was observed in 13 cases and the average time to recurrence was 15.78 months. The highest survival rate was observed in cervical cases. Comprehensive treatment may improve the survival rate of these patients, but the diagnosis of SCNCGT is challenging. SCNCGT is often diagnosed based on histopathological findings. Both morphological and immunohistochemical analyses are required for a differential diagnosis of SCNCGT. Immunohistochemical analyses must indicate positivity for at least one neuroendocrine marker to verify that the tumor cells have neuroendocrine functions. NSE is highly expressed in neuroendocrine tumors, ${ }^{2,15}$ however, despite its high sensitivity, specificity for this marker is poor. Previous studies have reported higher specificity for synaptophysin and chromogranin A than for NSE, albeit with lower sensitivity. ${ }^{2}$ Studies of small-cell carcinoma affecting various organs demonstrated that the positive rate for CD56 can aid in the pathological diagnosis of small-cell carcinoma. ${ }^{6}$

The NSE levels significantly increased, suggesting that NSE can aid in the differentiation of neuroendocrine small-cell carcinoma from other pathological types of cancer. However, given that neuroendocrine small-cell carcinoma is very rare, none of the reported cases of ovarian cancer or endometrial carcinoma involved NSE testing prior to diagnosis. In our study, the seven patients with SCNCC underwent assessments of NSE levels prior to surgery. The NSE levels ranged from 11.15 to $370 \mathrm{ng} /$ $\mathrm{mL}$ and increases in NSE levels varied among patients. These results highlight the involvement of NSE in smallcell carcinoma, as well as its potential as a marker of reproductive system malignancies. Such findings may aid in predicting the occurrence of small-cell carcinoma and in 
A

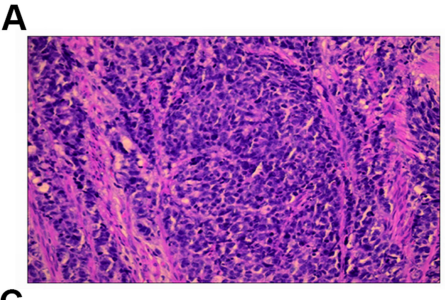

C

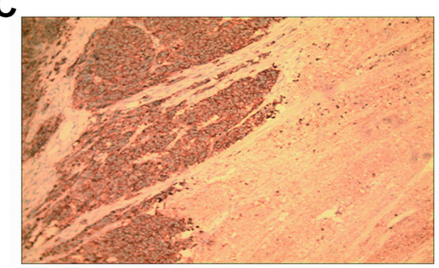

E

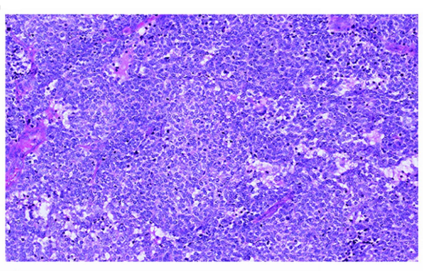

G

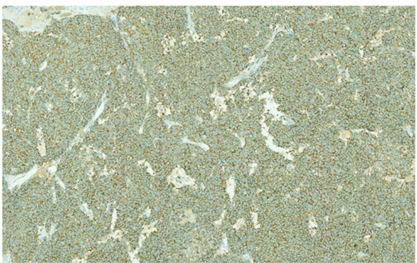

I

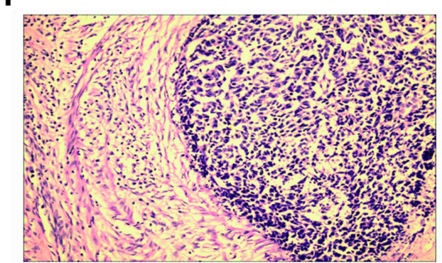

K

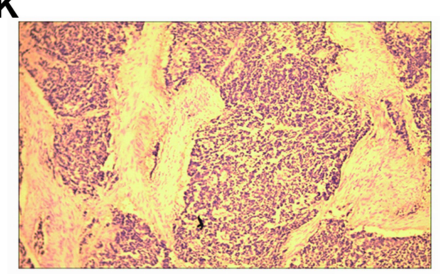

B

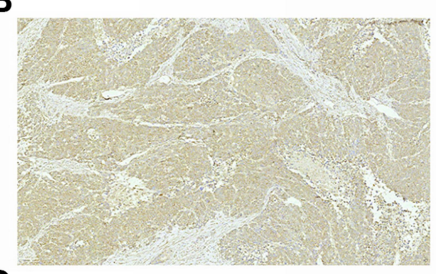

D

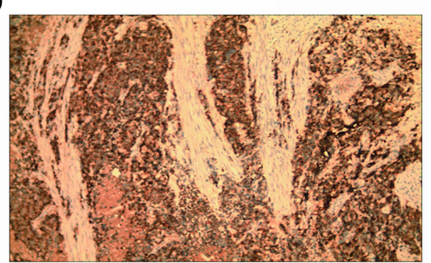

F

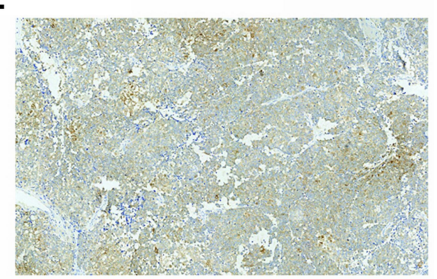

H

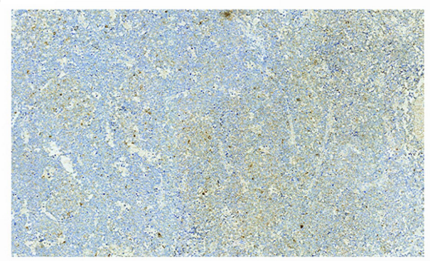

J
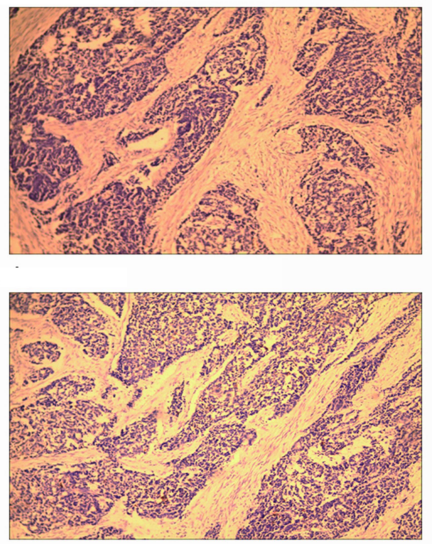

Figure I Pathological staining images for small-cell neuroendocrine carcinoma of the gynecologic tract (SCNCGT). (A) The small-cell neuroendocrine carcinoma of the endometrium (SCNCE) was characterized by small cells with scant cytoplasm (hematoxylin and eosin stain (H\&E); magnification $\times 200)$. (B) The SCNCE was positive for chromogranin A and neuron-specific enolase (H\&E; original magnification, $\times 100)$. (C) The SCNCE was positive for synaptophysin (H\&E; original magnification, $\times 100)$. (D) The SCNCE was positive for chromogranin A (H\&E; original magnification, $\times 100)$. (E) The small-cell neuroendocrine carcinoma of the ovary (SCNCO) was characterized by small cells with scant cytoplasm (H\&E; magnification $\times 200)$. (F) The SCNCO was positive for neuron-specific enolase (H\&E; original magnification, $\times 100)$. (G) The SCNCO was positive for synaptophysin (H\&E; original magnification, $\times 100)$. (H) The SCNCO was positive for chromogranin A (H\&E; original magnification, $\times 100)$. (I) The small-cell neuroendocrine carcinoma of the cervix (SCNCC) was characterized by small cells with scant cytoplasm (H\&E; magnification $\times 200)$. (J) The SCNCC was positive for neuron-specific enolase (H\&E; original magnification, $\times 100)$. (K) The SCNCC was positive for synaptophysin (H\&E; original magnification, $\times 100)$. (L) The SCNCC was positive for chromogranin $\mathrm{A}(\mathrm{H} \& \mathrm{E}$; original magnification, $\times 100)$.

establishing reference standards for prognosis and recurrence. Inzani et $\mathrm{al}^{16}$ recently identified a new marker for gynecological neuroendocrine cancer based on somatostatin receptor subtypes (SST2-SST5). Their findings indicate that the expression of SST2 and SST5 may support a role for somatostatin analogues (SSAs) in the 
Table 3 Treatment and Recurrence of Small-Cell Neuroendocrine Carcinoma of Gynecologic Tract for 34 Cases

\begin{tabular}{|c|c|c|c|c|c|c|c|c|c|}
\hline Case & Stage & Pre-OP & Operation & Adjuvant Therapy & Recurrence & $\begin{array}{l}\text { Interval } \\
\text { (Months) }\end{array}$ & Location & DFS & os \\
\hline \multicolumn{10}{|c|}{ SCNCE } \\
\hline 1 & IA & $N$ & $\mathrm{EFH}+\mathrm{Ad}$ & $4 \mathrm{EP}$ & $\mathrm{N}$ & & & 26 & 26 \\
\hline 2 & IA & $N$ & $\mathrm{EFH}+\mathrm{Ad}$ & $4 \mathrm{EP}$ & $Y$ & 6 & $\begin{array}{l}\text { Pelvic } \\
\text { cavity }\end{array}$ & 6 & 8 \\
\hline 3 & IB & $N$ & $\mathrm{EFH}+\mathrm{Ad}+\mathrm{LN}+\mathrm{PaLN}$ & $3 \mathrm{EP}$ & $Y$ & 5 & $\begin{array}{l}\text { Pelvic } \\
\text { cavity }\end{array}$ & 5 & 12 \\
\hline 4 & IIA & $N$ & $\mathrm{EFH}+\mathrm{Ad}+\mathrm{LN}+\mathrm{PaLN}$ & $4 \mathrm{EP}$ & $\mathrm{Y}$ & 21 & $\begin{array}{l}\text { Pelvic } \\
\text { cavity }\end{array}$ & 21 & 24 \\
\hline 5 & IIIC & $N$ & CS & $6 \mathrm{EP}$ & $N$ & & & 26 & 26 \\
\hline 6 & IIIC & $N$ & CS & $6 \mathrm{EP}$ & $Y$ & 26 & $\begin{array}{l}\text { Brain and } \\
\text { Bone }\end{array}$ & 26 & 34 \\
\hline 7 & IVB & $N$ & CS & I EP & $Y$ & I & $\begin{array}{l}\text { Pelvic } \\
\text { cavity }\end{array}$ & I & 3 \\
\hline \multicolumn{10}{|c|}{ SCNCO } \\
\hline 8 & IA & $N$ & UAd & $6 \mathrm{EP}$ & $\mathrm{N}$ & 7 & & 7 & 11 \\
\hline 9 & IA & $N$ & SS & $6 \mathrm{EP}$ & $Y$ & 30 & $\begin{array}{l}\text { Pelvic } \\
\text { cavity }\end{array}$ & 30 & 36 \\
\hline 10 & IIA & $N$ & SS & $6 \mathrm{EP}$ & $N$ & & & 16 & 16 \\
\hline II & IIIC & $N$ & Induced +CS & $4 \mathrm{EP}$ & $N$ & 16 & $\begin{array}{l}\text { Pelvic } \\
\text { cavity }\end{array}$ & 8 & 24 \\
\hline 12 & IIIC & $N$ & CS & 6 BEP & $N$ & & & 18 & 18 \\
\hline 13 & IIIC & $N$ & CS & 6 BEP & Y & 21 & Bone & 21 & 26 \\
\hline 14 & IVB & $N$ & NBAC & I TC+ | BEP & Y & 3 & $\begin{array}{l}\text { Whole } \\
\text { body }\end{array}$ & 3 & 4 \\
\hline \multicolumn{10}{|c|}{ SCNCC } \\
\hline 15 & $|\mathrm{~B}|$ & $N$ & $\mathrm{RH}+\mathrm{LN}+\mathrm{DOS}$ & EP 2 cycles & Y & & Lung & 22 & 30 \\
\hline 16 & $|\mathrm{~B}|$ & $N$ & $\mathrm{RH}+\mathrm{LN}+\mathrm{Ad}$ & TC 6 cycles & $\mathrm{N}$ & & & 31 & 31 \\
\hline 17 & $|\mathrm{~B}|$ & $N$ & $\mathrm{RH}+\mathrm{LN}+\mathrm{Ad}$ & TC 6 cycles & $\mathrm{N}$ & & & 52 & 52 \\
\hline 18 & IB2 & $N$ & $\mathrm{RH}+\mathrm{LN}+\mathrm{DOS}$ & TC 3 cycles & $\mathrm{N}$ & & & 22 & 22 \\
\hline 19 & IB2 & $N$ & $\mathrm{RH}+\mathrm{LN}+\mathrm{Ad}$ & TC 3 cycles + EP 2 cycles & $N$ & & & 7 & 12 \\
\hline 20 & IB3 & $N$ & $R H+L V+A d+P a L N$ & EP 2 cycles & $Y$ & & Brain & 12 & 12 \\
\hline 21 & IB3 & $N$ & $\mathrm{RH}+\mathrm{LN}+\mathrm{DOS}+\mathrm{PaLN}$ & TC 3 cycles + RT & $N$ & & & 6 & 6 \\
\hline 22 & IB3 & $N$ & $\mathrm{RH}+\mathrm{LN}+\mathrm{DOS}+\mathrm{PaLN}$ & TC 3 cycles + RT & $\mathrm{N}$ & & & 15 & 19 \\
\hline 23 & IB3 & $N$ & $\mathrm{RH}+\mathrm{LN}+\mathrm{DOS}$ & EP 2 cycles & $\mathrm{N}$ & & & 12 & 16 \\
\hline 24 & IIA & $N$ & $R H+L N+A d+P a L N$ & $\begin{array}{l}\text { TC } 3 \text { cycles + biological } \\
\text { treatment }\end{array}$ & $\mathrm{N}$ & & & 34 & 34 \\
\hline
\end{tabular}


Table 3 (Continued).

\begin{tabular}{|c|c|c|c|c|c|c|c|c|c|}
\hline Case & Stage & Pre-OP & Operation & Adjuvant Therapy & Recurrence & $\begin{array}{l}\text { Interval } \\
\text { (Months) }\end{array}$ & Location & DFS & os \\
\hline 25 & $\| \mathrm{A}$ & $\mathrm{N}$ & $\mathrm{RH}+\mathrm{LN}+\mathrm{Ad}+\mathrm{PaLN}$ & EP 2 cycles & $\mathrm{N}$ & & & 48 & 48 \\
\hline 26 & IIA & $\mathrm{N}$ & $\mathrm{RH}+\mathrm{LN}+\mathrm{DOS}+\mathrm{PaLN}$ & EP 2 cycles & $\mathrm{N}$ & & & 18 & 18 \\
\hline 27 & $\| \mathrm{A}$ & TP 3 cycle & $\mathrm{RH}+\mathrm{LN}+\mathrm{Ad}+\mathrm{PaLN}$ & TP 2 cycles & $\mathrm{N}$ & & & 16 & 21 \\
\hline 28 & $\| \mathrm{A}$ & $\mathrm{N}$ & $\mathrm{RH}+\mathrm{LN}+\mathrm{Ad}+\mathrm{PaLN}$ & EP 3 cycles & $\mathrm{N}$ & & & 21 & 34 \\
\hline 29 & IIA & $\mathrm{N}$ & $\mathrm{RH}+\mathrm{LN}+\mathrm{Ad}+\mathrm{PaLN}$ & TP 2 cycles + RT & $Y$ & & Brain & 54 & 54 \\
\hline 30 & $\| \mathrm{A}$ & $\mathrm{N}$ & $\mathrm{RH}+\mathrm{LN}+\mathrm{DOS}+\mathrm{PaLN}$ & TC 3 cycles & $\mathrm{N}$ & & & 66 & 66 \\
\hline 31 & IIB & $\mathrm{N}$ & RTC & & $\mathrm{N}$ & & & 12 & 12 \\
\hline 32 & Illb & $\mathrm{N}$ & RTC & & $\mathrm{N}$ & & & 21 & 21 \\
\hline 33 & IVB & $\mathrm{N}$ & RTC & & $Y$ & & $\begin{array}{l}\text { Whole } \\
\text { body }\end{array}$ & 6 & 7 \\
\hline 34 & IVB & $\mathrm{N}$ & RTC & & $\mathrm{N}$ & & & 5 & 5 \\
\hline
\end{tabular}

Abbreviations: OP, operation; DFS, disease-free survival; OS, overall survival; SCNCE, small-cell neuroendocrine carcinoma of endometrium; RH, radical hysterectomy; EFH, extra-fascial hysterectomy; Ad, adnexectomy; EP, etoposide/cisplatin; N, no; Y, yes; LN, lymph node; Pa-LN, para-aortic lymph node resection; CS, cytoreductive surgery; NBAC, needle biopsy of the abdominal cavity; SCNCO, small-cell neuroendocrine carcinoma of ovary; UAd, unilateral adnexectomy; SS, staged surgery; TC, paclitaxel/carboplatin; BEP, bleomycin/etoposide/cisplatin; SCNCC, small-cell neuroendocrine carcinoma of cervix; RH, radical hysterectomy; DOS, double ovarian suspension; SCNCGT, small-cell neuroendocrine carcinoma of gynecologic tract; LND, lymph node dissection; RTC, radiotherapy concurrent chemotherapy; RT, radiotherapy.

diagnosis and therapy of patients with cervical neuroendocrine carcinoma.

Previous studies have reported increases in serum CA125 levels in patients with SCNCO. ${ }^{17}$ Among the seven patients with SCNCO in our study, preoperative increases in CA125 levels were observed only in two cases (highest: $970 \mathrm{U} / \mathrm{mL}$ ), while the remaining values were within the normal range. In addition, CA125 levels again increased to $221 \mathrm{U} / \mathrm{mL}$ following tumor recurrence in the patient with the highest CA125 levels. In the 20 patients with SCNCC, the SCC levels in the inguinal lymph nodes were all within the normal range. CA125 levels were also within the normal range in patients with SCNCE. Overall, these findings indicate that serum CA125 levels can be used to monitor the treatment and progression of SCNCO. In the present study, SCNCGT was staged in accordance with the 2009 FIGO criteria. However, given its rarity, currently there is no standardized treatment plan. A comprehensive treatment plan combining surgery, radiotherapy, and chemotherapy is selected mainly based on the primary site of the tumor. The choice of chemotherapy regimens is usually made with reference to those utilized in patients with small-cell lung cancer or gynecological tumors. ${ }^{18,19}$
All seven patients with SCNCE in our study underwent the corresponding surgical procedures for the staging of endometrial adenocarcinoma. Because they were all postmenopausal, patients with stage I or II malignancies underwent $\mathrm{EFH}+\mathrm{AD}+\mathrm{LN}+\mathrm{Pa}-\mathrm{LN}$. CS was performed in patients with stage III or IV malignancies, followed by one to six cycles of postoperative EP. Among the seven patients with SCNCE, five experienced disease progression. The shortest time to disease progression was only 0.5 months, and the shortest OS time was 3 months. However, Sawada et $\mathrm{al}^{20}$ reported a survival time of 12 years in a patient with advanced SCNCE with liver and brain metastases; the patient underwent CS and metastasis resection. After the operation, the patient was treated with irinotecan hydrochloride combined with cisplatin chemotherapy, adjuvant RTC, and postoperative use of hydrochloric acid. Viau et $\mathrm{al}^{21}$ further reported the case of a patient diagnosed with stage IV SCNCE who remains disease-free 5 years after treatment with surgery, chemotherapy (EP), and RTC. These findings highlight the importance of comprehensive treatment for improving the prognosis for patients with SCNCE. Thus, further studies are required to develop appropriate standards for comprehensive treatment. None of seven patients with SCNCE in 
A

\section{Disease-free Survival}
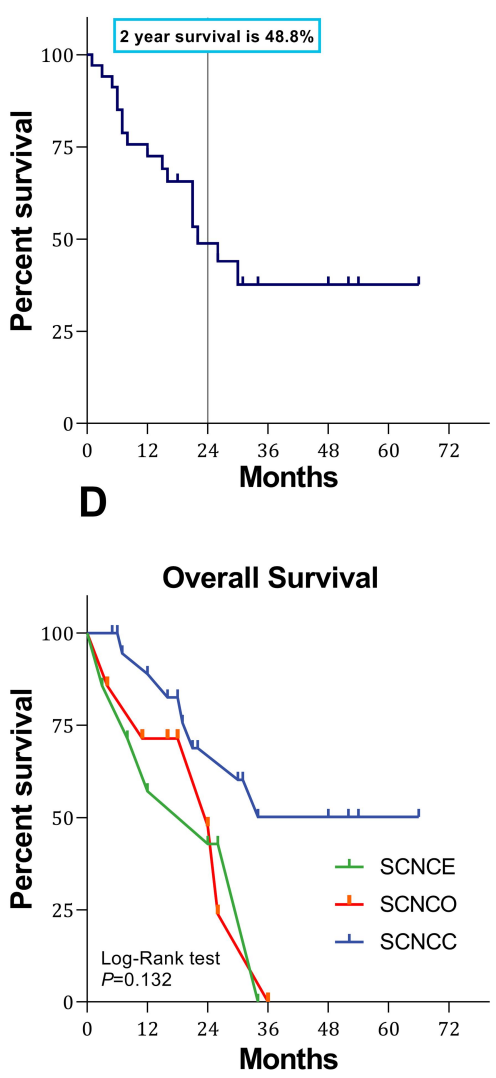

B

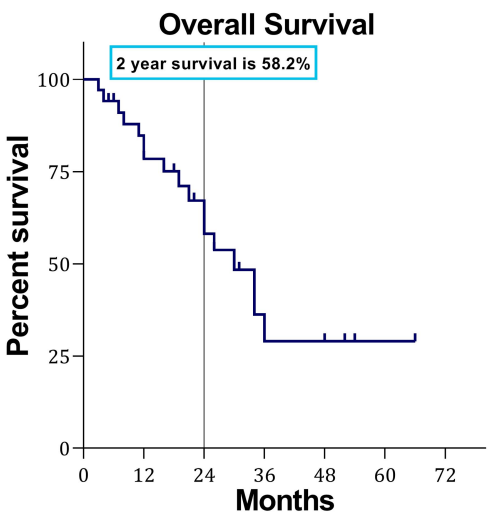

E

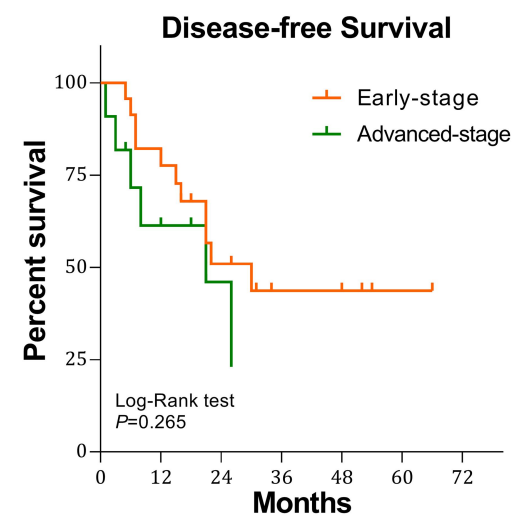

C

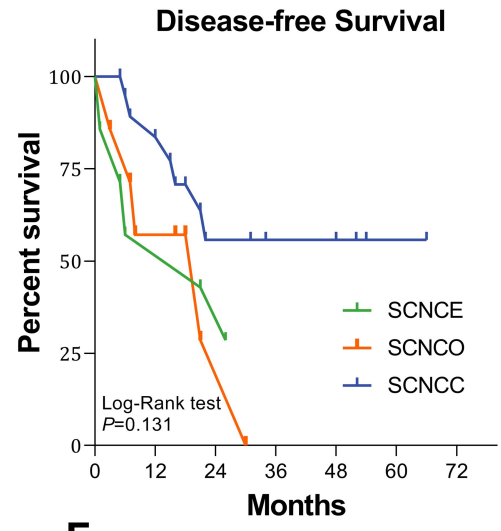

$\mathbf{F}$

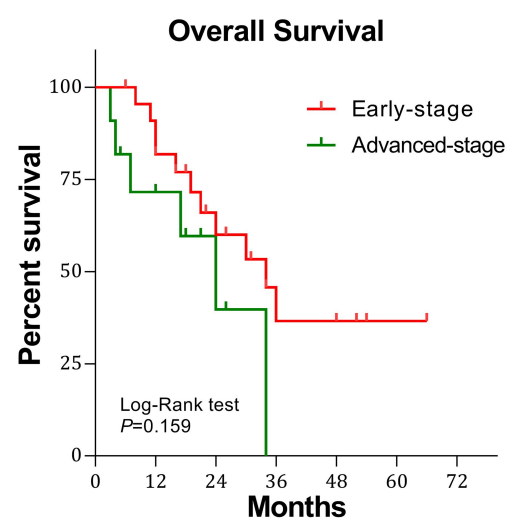

Figure 2 Survival curves of all 34 patients with small-cell neuroendocrine carcinoma of the gynecologic tract (SCNCGT). (A) Kaplan-Meier curve showing the disease-free survival (DFS) rate in patients with SCNCGT. (B) Kaplan-Meier curve showing the overall survival (OS) rate in patients with SCNCGT (C) Kaplan-Meier curve showing the DFS rate in patients with small-cell neuroendocrine carcinoma of the endometrium (SCNCE), ovary (SCNCO), and cervix (SCNCC), respectively. Log rank test resulted in $P=0.131$. (D) Kaplan-Meier curve showing the OS rate in patients with small-cell neuroendocrine carcinoma of the endometrium (SCNCE), ovary (SCNCO), and cervix (SCNCC), respectively. Log rank test resulted in $P=0.132$. (E) Kaplan-Meier curve showing the DFS rate in patients with early-stage and advanced-stage disease, respectively. Log rank test resulted in $P=0.265$. (F) Kaplan-Meier curve showing the OS rate in patients with early-stage and advanced-stage groups, respectively. Log rank test resulted in $\mathrm{P}=0.159$.

our study received radiotherapy. Among these patients, those with stage IV SCNCE relapsed for a short period of time and refused further treatment. The remaining six patients did not receive radiotherapy, constituting a limitation in our treatment of endometrial small-cell carcinoma. These findings may provide new insights into the treatment of small-cell carcinoma, which may in turn help to prolong patient survival.

SCNCO is mainly treated via surgery and adjuvant chemotherapy. Patients with stage I and II malignancies typically undergo ovarian cancer staging surgery, while those with stage III and IV malignancies undergo CS for ovarian cancer. The currently recommended chemotherapy regimen is a combination of platinum and etoposide administered postoperatively. ${ }^{22,23}$ Nasioudis et $\mathrm{al}^{22}$ reported that 5-year OS rates for stage I, II, III, and IV disease were $48.6 \%, 30.7 \%, 18 \%$, and $12.3 \%$ among 469 women with SCNCO, respectively. Younger age and earlier chemotherapy were associated with higher OS rates. A multivariate analysis revealed that an earlier disease stage and the use of CT were associated with lower mortality, while the patient's age and the performance of lymph node dissection were not. In our study, the seven patients with SCNCO underwent three to six cycles of adjuvant chemotherapy. However, such treatment was not very effective. Three patients developed pelvic metastases, and one patient with stage IIA disease survived only 24 months. Moreover, the median OS time was only 19.28 months (range: 4-36 months), and the median duration of DFS was only 14.71 months (range: 3-30 months). Further studies are required to determine the most appropriate chemotherapy regimen for patients with SCNCO. 
Gadducci et $\mathrm{al}^{24}$ retrospectively analyzed several studies related to the treatment of SCNEC, which requires multimodal therapy even in early stages of the disease due to the risk of developing distant metastases. Such therapy usually consists of RH followed by adjuvant chemotherapy or concurrent chemoradiation for early-stage or locally advanced disease. SCNCC is highly invasive. When compared with traditional cervical squamous cell carcinoma, SCNCC is associated with increases in vascular invasion, lymph node metastasis, and distant metastasis. ${ }^{25-28}$ Of our 20 patients with SCNCC (30\%), six exhibited vascular metastases, while eight (40\%) exhibited pelvic lymph node metastases. A previous study including 71 patients across 25 medical centers in Japan ${ }^{11}$ examined the effects of postoperative adjuvant treatment (chemotherapy with platinum drugs vs non-chemotherapy). The authors reported significantly better progression-free survival (PFS) and OS in the postoperative chemotherapy group than in the non-chemotherapy group. In a study of 40 patients with SCNCC, $\mathrm{Xu}$ et $\mathrm{al}^{29}$ reported that FIGO stage and radiotherapy (RT) were associated with poor survival. However, other studies have indicated that radical surgery can prolong survival. In a meta-analysis of 1901 patients, RT was also associated with poor survival, while chemotherapy was associated with prolonged survival, similar to findings observed among patients with small-cell lung cancer. Lin et $\mathrm{al}^{30}$ reported that brachytherapy may improve OS for stage II-IVA SCNCC and that the treatment may be underutilized. Although first-line treatment for SCNCC includes surgery and adjuvant chemotherapy, these findings indicate that clinicians should be cautious when selecting RT. Given our small sample size, further studies are required to determine the influence of RT on survival in this patient population. The biological characteristics of SCNCGT are unique. Such tumors are characterized by diffuse interstitial infiltration, and 40 $50 \%$ of SCNCGTs are associated with early metastasis/ lymph node enlargement, which can easily manifest as distant metastasis to the liver, lungs, bone, brain, and even the skin. ${ }^{11}$ In our study, $44.1 \%$ of patients had lymph node metastases, $32.3 \%$ had vascular metastases, and 17 patients died. The average survival time was 23.18 months. There were 13 cases of recurrence, resulting in a recurrence rate of $38.24 \%$. The earliest time to recurrence following surgery was 0.5 months. DFS ranged from 1 to 66 months, and the median PFS was 20 months. Early- and late-stage disease were observed in $70.6 \%$ and $29.4 \%$ of cases, respectively. However, 11 patients with early-stage disease died, and seven experienced recurrence. These findings indicate that the rates of metastasis and recurrence are high, even among patients with earlystage disease.

The strength of the present study is that we retrospectively analyzed the clinical/pathological features of SCNCGT and relevant treatment methods in a comprehensive manner. However, the study is limited by its single-center design and small sample size. Thus, further studies involving larger samples of patients across multiple centers are required to verify our findings.

Prognosis remains poor for patients with SCNCGT. Considering that it is relatively rare in clinical practice this may be related to the late detection of the disease; standardized methods for early detection and treatment may help to improve prognosis. Our novel findings indicate that staging is the most important prognostic factor for patients with SCNCE, SCNCC, and SCNCO. Among them, SCNCE has the shortest OS and SCNCO has the shortest PFS. Early detection and comprehensive treatment may help improve the prognosis. Due to its rarity, most previous research related to SCNCGT has been published in the form of case reports. Additional studies including a large number of patients across multiple sites are required to elucidate the biological behavior of the disease and explore the most suitable treatment methods for improving prognosis among affected patients. Future studies should also aim to investigate the value of targeted gene therapy, methods for improving preoperative diagnosis, and the most appropriate treatment strategies for patients with SCNCGT.

\section{Abbreviations}

$\mathrm{AD}$, adnexectomy; $\mathrm{BEP}$, bleomycin + etoposide + cisplatin; $\mathrm{CK}$, creatine kinase; CS, cytoreductive surgery; DFS, diseasefree survival; EFH, extra-fascial hysterectomy; EP, etoposide + cisplatin; FIGO, Federation of Gynecology and Obstetrics; LN, pelvic lymph node dissection; NSE, neuron-specific enolase; OS, overall survival; Pa-LN, Para-aortic lymph node resection; PFS, progression-free survival; RH, radical hysterectomy; RTC, radiotherapy and chemotherapy; SCC, Squamous cell carcinoma; SCNCC, small-cell neuroendocrine carcinoma of the cervix; SCNCE, small-cell neuroendocrine carcinoma of the endometrium; SCNCGT, small-cell neuroendocrine carcinoma of the gynecologic tract; SCNCO, smallcell neuroendocrine carcinoma of the ovary; SSAs, somatostatin analogues; TC, paclitaxel/carboplatin; TP, docetaxel + cisplatin. 


\section{Ethics Approval and Informed Consent}

This retrospective study was approved by the Ethics Committee of ShengJing Hospital of China Medical University. All patients provided informed written consent. This study was conducted in accordance with the Declaration of Helsinki.

\section{Consent for Publication}

All listed authors have actively participated in the study and have read and approved the submitted manuscript.

\section{Funding}

No financial support was received for this work.

\section{Disclosure}

The authors report no conflicts of interest in this work.

\section{References}

1. Motiwala H, Bansal I, Goyal P, et al. Do we really care about incidental lung nodules? Review of atypical lung carcinoid and a proposal for systematic patient follow up. Transl Lung Cancer Res. 2017;6:387-392. doi:10.21037/tlcr.2017.05.05

2. Katahira A, Akahira J, Niikura H, et al. Small cell carcinoma of the endometrium report of three cases and literature review. Int $J$ Gynecol Cancer. 2004;14:1018-1023. doi:10.1136/ijgc-00009577-20040900000041

3. Atienza-Amores M, Guerini-Rocco E, Soslow RA, Park KJ, Weigelt B. Small cell carcinoma of the gynecologic tract: a multifaceted spectrum of lesions. Gynecol Oncol. 2014;134:410-418. doi:10.1016/j.ygyno.2014.05.017

4. Cohen JG, Chan JK, Kapp DS. The management of small-cell carcinomas of the gynecologic tract. Curr Opin Oncol. 2012;24:572-579. doi:10.1097/CCO.0b013e3283565ed6

5. Kuji S, Hirashima Y, Nakayama H, et al. Diagnosis, clinicopathologic features, treatment, and prognosis of small cell carcinoma of the uterine cervix; Kansai Clinical Oncology Group/Intergroup study in Japan. Gynecol Oncol. 2013;129:522-527. doi:10.1016/j. ygyno.2013.02.025

6. Crowder S, Tuller E. Small cell carcinoma of the female genital tract. Semin Oncol. 2007;34:57-63. doi:10.1053/j.seminoncol.2006.10.028

7. Howitt BE, Kelly P, McCluggage WG. Pathology of neuroendocrine tumours of the female genital tract. Curr Oncol Rep. 2017;19:59. doi:10.1007/s11912-017-0617-2

8. Patibandla JR, Fehniger JE, Levine DA, Jelinic P. Small cell cancers of the female genital tract: molecular and clinical aspects. Gynecol Oncol. 2018;149:420-427. doi:10.1016/j.ygyno.2018.02.004

9. Lopes Dias J, Cunha TM, Gomes FV, Callé C, Félix A. Neuroendocrine tumours of the female genital tract: a case-based imaging review with pathological correlation. Insights Imaging. 2015;6:43-52. doi:10.1007/s13244-014-0378-5

10. FIGO Committee on Gynecologic Oncology. FIGO staging for carcinoma of the vulva, cervix, and corpus uteri. Int J Gynaecol Obstet. 2014;125:97-98. doi:10.1016/j.ijgo.2014.02.003

11. Meinhold-Heerlein I, Fotopoulou C, Harter P, et al. The new WHO classification of ovarian, fallopian tube, and primary peritoneal cancer and its clinical implications. Arch Gynecol Obstet. 2016;293:695-700. doi:10.1007/s00404-016-4035-8
12. Bhatla N, Denny L. FIGO Cancer Report. Int $J$ Gynecol Obstet. 2018;143:22-36. doi:10.1002/ijgo.12611

13. Bhatla N, Berek JS, Cuello Fredes M. Corrigendum to "Revised FIGO staging for carcinoma of the cervix uteri". Int $J$ Gynecol Obstet. 2019;145(1):129-135. doi:10.1002/ijgo.12749

14. Kurman RJCM, Herrington CS, Young RH. World Health Organization Classification of Tumours of Female Reproductive Organs. Lyon: IARC Press; 2014.

15. Matsumoto H, Takai N, Nasu K, Narahara H. Small cell carcinoma of the endometrium: a report of two cases. J Oset Gynaecol Res. 2011;37:1739-1743. doi:10.1111/j.1447-0756.2011.01593.x

16. Inzani F, Santoro A, Angelico G, et al. Neuroendocrine carcinoma of the uterine cervix: a clinicopathologic and immunohistochemical study with focus on novel markers (Sst2-Sst5). Cancers. 2020;12:1211. doi:10.3390/cancers12051211

17. Jamy O, Yaghmour G, Hare F, Martin MG. Population-based analysis of the clinical features of primary small cell carcinoma of the ovary. Anticancer Res. 2015;35:3091-3095.

18. He Y, Zhao H, Li XM, Yin CH, Wu YM. A clinical analysis of small-cell neuroendocrine carcinoma of the gynecologic tract: report of 20 cases. Arch Gynecol Obstet. 2019;299:543-549. doi:10.1007/ s00404-018-4960-9

19. Lee SW, Lim KT, Bae DS, et al. A multicenter study of the importance of systemic chemotherapy for patients with small-cell neuroendocrine carcinoma of the uterine cervix. Gynecol Obstet Invest. 2015;79:172-178. doi:10.1159/000367920

20. Sawada M, Matsuzaki S, Yoshino K, et al. Long-term survival in small-cell carcinoma of the endometrium with liver and brain metastases. Anticancer Drugs. 2016;27:138-143. doi:10.1097/ CAD.0000000000000289

21. Viau M, Baragar I, Altman AD. Long-term survival in a stage IV small cell carcinoma of the endometrium. Gynecol Oncol Rep. 2020;32:100580. doi:10.1016/j.gore.2020.100580

22. Nasioudis D, Chapman-Davis E, Frey MK, Caputo TA, Witkin SS, Holcomb K. Small cell carcinoma of the ovary: a rare tumor with a poor prognosis. Int J Gynecol Cancer. 2018;28:932-938. doi:10.1097/ IGC.0000000000001243

23. Reed NS, Pautier P, Åvall-lundqvist E, et al. Gynecologic Cancer InterGroup (GCIG) consensus review for ovarian small cell cancers. Int $J$ Gynecol Cancer. 2014;24:S30-34. doi:10.1097/ IGC. 0000000000000293

24. Gadducci A, Carinelli S, Aletti G. Neuroendrocrine tumors of the uterine cervix: a therapeutic challenge for gynecologic oncologists. Gynecol Oncol. 2017;144:637-646. doi:10.1016/j.ygyno.2016.12.003

25. Qin JC, Li L, Yang ZJ, Yao DS, Li F, Zhang JQ. Clinicopathologic analysis of small cell neuroendocrine carcinoma of the cervix. Chin J Obstet Gynecol. 2011;46:360-363.

26. Zivanovic O, Leitao MM Jr, Park KJ, et al. Small cell neuroendocrine carcinoma of the cervix: analysis of outcome, recurrence pattern and the impact of platinum-based combination chemotherapy. Gynecol Oncol. 2009;112:590-593. doi:10.1016/j.ygyno.2008.11.010

27. Tempfer CB, Tischoff I, Dogan A, et al. Neuroendocrine carcinoma of the cervix: a systematic review of the literature. BMC Cancer. 2018;18:530. doi:10.1186/s12885-018-4447-x

28. Zhang Q, Xiong Y, Ye J, Zhang L, Li L, Wei Q. Influence of clinicopathological characteristics and comprehensive treatment models on the prognosis of small cell carcinoma of the cervix: a systematic review and meta-analysis. PLoS One. 2018;13(4): e0192784. doi:10.1371/journal.pone.0192784

29. Xu F, Ma J, Yi H, et al. Clinicopathological aspects of small cell neuroendocrine carcinoma of the uterine cervix: a multicenter retrospective study and meta-analysis. Cell Physiol Biochem. 2018;50:1113-1122. doi: $10.1159 / 000494538$

30. Lin AJ, Hassanzadeh C, Markovina S, Schwarz J, Grigsby P. Brachytherapy and survival in small cell cancer of the cervix and uterus. Brachytherapy. 2019;18:163-170. doi:10.1016/j.brachy.2018.11.006 


\section{Publish your work in this journal}

Cancer Management and Research is an international, peer-reviewed open access journal focusing on cancer research and the optimal use of preventative and integrated treatment interventions to achieve improved outcomes, enhanced survival and quality of life for the cancer patient.

The manuscript management system is completely online and includes a very quick and fair peer-review system, which is all easy to use. Visit http://www.dovepress.com/testimonials.php to read real quotes from published authors.

Submit your manuscript here: https://www.dovepress.com/cancer-management-and-research-journa 